geomagnetic field, is very complex and the existing observational information is poor. Only occasional measurements have been made by rockets or, at the upper end of the altitude range, by a few satellites at perigee or during their decay phase. At an altitude of about $100 \mathrm{~km}$, the mean free path of the atmospheric gas is comparable to the typical satellite size. Above, molecular diffusion becomes predominant, i.e. the atmospheric constituents begin to separate from each other, and atomic oxygen becomes important. The turbulence typical of altitudes up to $100 \mathrm{~km}$ is no longer a dominant factor.

Among the principal scientific objectives for low altitude satellites, is the determination of the chemical composition of the neutral atmosphere, in the region where the vertical transport processes are important for the exchange of hydrogen (upwards) and nitrogen compounds (downwards). A number of different atomic and molecular species are present $\left(\mathrm{H}, \mathrm{H}_{2}, \mathrm{O}, \mathrm{O}_{2}, \mathrm{~N}_{2}\right.$, as well as traces of minor components, for example sulphur); these particles can combine with each other in a variety of ways, so that a large number of components exist, with a large spectrum of properties and lifetimes. Realistic modelling of the atmospheric composition and vertical distribution are in dire need of insitu mass spectrometric measurements. Also, chemical tracers inside large volumes and areas can be seeded in order to study the complex pattern of neutral atmospheric circulation.

Another kind of basic problem has to do with fluid dynamics: in particular, aerodynamic and heat transfer coefficients within a variety of conditions which cannot be obtained by current wind tunnel technology, because of the impossibility of making thermo-fluid-dynamic measurements in regimes where low Reynolds numbers are combined with high Mach numbers. Low altitude tethered satellites may also be used as a sort of "open wind tunnel", operating over time ranges much longer than any existing or proposed ground facility.

Also in the field of remote sensing, tethered satellites will prove invaluable for increasing the accuracy of real time cartographic maps from space.

\section{The ionized atmosphere}

In the same altitude range, the atmospheric components are subject to the ionizing action of ultraviolet solar radiation. The energy released in the process heats the gas to temperatures that increase as the altitude increases: electrons, positive ions and neutrals exhibit

\title{
Physics Education Counsellors for China
}

The European Physical Society has been invited by the Citizen Ambassador Program based in the USA to submit the name(s) of a leader to head an international delegation of professionals in physics education to China. The leader would be expected to participate in the development phase of the project that has been initiated by the Physical Society of the People's Republic of China. Responsibilities would include agreeing on the area of professional interest and selecting the delegation members. Expenses would be covered by the Program. No dates have yet been fixed for the delegation's visit, but a period in the early Autumn of this year or Spring of 1990 is projected. Qualified physicists interested in this project should contact the EPS Secretariat in Geneva for further details.

different vertical profiles of temperature, because thermal exchange decreases with altitude owing to the fast transition from a regime of high collisional rate between neutrals and ions to one of vanishing rate. At lower altitudes, the ions are carried along by neutral winds, while a few tens of $\mathrm{km}$ above, electric field drift motions dominate. Global motions of the ionized components embedded in the neutral gas give rise in the presence of the geomagnetic field to electrical currents (the so-called dynamo currents), which are the main source of geomagnetic variations on the ground, either regular (in particular diurnal) or irregular, as well as of Joule heating. These currents, which are essentially ionospheric at low and middle latitudes, are indeed the low latitude aspect of the complicated current circulation pattern taking place at higher latitudes. In the polar caps, owing to the high conductivity along the nearly vertical geomagnetic field lines, a system of aligned currents, called Birkeland currents, builds up connecting the lower atmosphere and ionosphere to the external magnetospheric regions, where the impinging solar wind delivers energy to the magnetosphere. This current pattern at high latitudes and in the polar caps is subject to large perturbations, in particular during geomagnetic storms and in the not infrequent event of bombardment by high energy protons from the Sun.

\section{Physica
Scripta}

An international journal for experimental and theoretical physics

Vol. T25 1989

\section{Proceedings of the 8th General Conference of the EPS Condensed Matter Division}

\section{Budapest, 6-9 A pril 1988}

Edited by: F. Beleznay, J. Kollár, I. Kovács, N. Kroó, N. Menyhárd

In addition to plenary lectures and all the individual invited talks the editors have selected contributions to the Symposia under the headings:

$\mathrm{X}$-ray and electron microscopic structural analysis; Charge density waves in solids; Localization; High $\mathrm{T}_{\mathrm{c}}$ superconductivity, Liquid crystals, Quantum tunnelling in complex systems; Transition metals in semiconductors; Spin glass models and memory; Electron spectroscopy; Novel physics of quantum wells and superlattices; Light scattering; Topics in magnetism; Chaos, fractals, turbulence; Applications; Large scale computation; Hydrogen and bonding; Liquids; Surface physics.

376 pages in A4 format covering almost 70 talks.

Orders to Physics Scripta at the address shown below; Price: SKR 900.- or \$US 160.- 